2010

\title{
Acute Exercise Is Associated With Greater Nighttime Blood Pressure Dipping In Women Classified As Non-dippers
}

Kimberly P. Ferrante

William \& Mary, kpferr@wm.edu

David G. Wilson

William \& Mary

Danielle D. Jones

William \& Mary

Erica M. Jackson

William \& Mary

Follow this and additional works at: https://scholarworks.wm.edu/aspubs

\section{Recommended Citation}

Ferrante, K. P., Wilson, D. G., Jones, D. D., \& Jackson, E. M. (2010). Acute Exercise Is Associated With Greater Nighttime Blood Pressure Dipping In Women Classified As Nondippers: 1961Board\# 90 June 3 9: 00 AM-10: 30 AM. Medicine \& Science in Sports \& Exercise, 42(5), 459. 
PURPOSE: To increase medical students' knowledge of physical activity guidelines and provide strategies to improve their exercise counseling techniques. METHODS: During an Ambulatory Practice Clerkship, third-year medical students participated in a 1.5 hour small group didactic session. Topics discussed included physical activity guidelines; definitions of exercise intensity; benefits of physical activity; strategies for identifying patient barriers to physical activity and developing strategies to assist patients in overcoming them, and exercise testing guidelines. Students were provided with Exercise is Medicine $\mathrm{TM}$ materials from the initiative's website. At the conclusion of the session, students completed an evaluation form that assessed the (1) value of the session to them personally, and (2) relevance to their work with future patients on a scale of $1=$ Poor to $5=$ Excellent. Students were also instructed to list one new fact or strategy they learned.

RESULTS: Over the 2008-2009 academic year, 93 students completed the small group didactic sessions. For each of the topics the personal value and patient relevance evaluation scores were as follows: physical activity guidelines (4.1 personal, 4.7 patient), definitions of exercise intensity (4.0, 4.5), benefits of physical activity $(3.8,4.4)$, strategies for barriers $(3.7,4.4)$, and exercise testing recommendations $(3.7,4.3)$. When identifying a new fact or strategy learned, $42 \%$ of student responses related to physical activity guidelines, $23 \%$ to strategies for identifying and overcoming physical activity barriers, $14 \%$ to exercise testing recommendations, $12 \%$ to definitions of exercise intensity, and $9 \%$ to the benefits of physical activity. On the Ambulatory Practice Clerkship final exam, $61 \%$ of students were able to correctly identify the current physical activity guidelines.

CONCLUSION: Medical students who participated in small group sessions that incorporated Exercise is Medicine TM principles found the topics relevant to them personally and even more relevant to their patients. Content related to physical activity guidelines was the area in which most medical students reported learning a new fact or strategy. The results illustrate the need for ongoing incorporation of the Exercise is Medicine ${ }^{\mathrm{TM}}$ initiative into medical education. Further research should be done to assess the long-term impact of the didactic sessions.

1960 Board \#89 June $3 \quad 9: 00$ AM - 10:30 AM

The Influence of Exercise on Neuromuscular Function in Adults on the Autism Spectrum

Brian D. Hand, Jill A. Bush, FACSM, Lisa Crabtree, Rebecca Newhouse, Lisa Fagan, Raymond Stinar. Towson University, Towson, MD. Email: BHand@towson.edu

(No disclosure reported)

Autism spectrum disorders cause a primary impairment in social interaction, but also affect neuromuscular function. This can impact participation in physical activity and affect quality of life. Exercise has been shown to have an impact on neuromuscular function in various populations.

PURPOSE: To determine the effect of exercise on neuromuscular function as assessed by balance and gait, in young adults on the autism spectrum who attend the Towson University Center for Adults with Autism Spectrum Disorders Wellness Program.

METHODS: Balance and gait were assessed before and after 6 weeks of participation in aerobic and strength and conditioning exercises. The program consisted of 2 days per week of exercises for cardiorespiratory fitness, muscular strength, muscular endurance, flexibility, and balance for 1.5 hours, including a group warm-up and cooldown that promoted social interaction and provided healthy lifestyle education. Participants were male ( $\mathrm{n}=7$ ) and female ( $\mathrm{n}=1$ ) adults (20-34 yrs) from the community who are on the autism spectrum. Changes in balance and gait were assessed using the Tinetti Performance Oriented Mobility Assessment (POMA). Paired t-tests were used to test for significant differences in changes in balance, in gait at normal pace, and in gait at a rapid pace before and after 6 weeks of participation in the Towson University Autism Wellness Program.

RESULTS: There was a significantly greater increase in normal gait scores after participation in the program compared with before $(10.25 \pm 1.38$ vs. $8.38 \pm 2.50$ $\mathrm{P}=0.008)$. Also, there was a significantly greater increase in rapid pace gait scores after participation in the Towson University Autism Wellness Program compared with before $(10.38 \pm 1.69$ vs. $8.00 \pm 2.10, P=0.001)$. There was no significant difference in balance scores before and after 6 weeks of participation. CONCLUSIONS: These preliminary results suggest that in young adults on the autism spectrum that exercise at least 2 days per week for 6 weeks may improve neuromuscular function as assessed by normal and rapid pace gait.

\section{Board \#90 June $3 \quad 9: 00$ AM - 10:30 AM}

Acute Exercise Is Associated With Greater Nighttime Blood Pressure Dipping In Women Classified As Non-dippers

Kimberly P. Ferrante, David G. Wilson, Danielle D. Jones, Erica M. Jackson. College of William and Mary, Williamsburg, VA.

Email: kpferr@wm.edu

(No disclosure reported)

Acute exercise has been shown to improve the non-dipping (ND) blood pressure pattern in hypertensive individuals. Prehypertensive individuals have increased risk for developing hypertension and for having a ND pattern.

PURPOSE: To assess whether acute exercise affects the non-dipping blood pressure of prehypertensive individuals.

METHODS: We assessed 24-hour blood pressure patterns in low to moderately active normotensive $(n=8)$ and prehypertensive $(n=22)$ women. Ten of the prehypertensive had a ND pattern for mean arterial pressure (MAP). Participants wore an ambulatory blood pressure monitor on two days separated by one week. In the first session, participants wore the monitor on a non-exercise weekday, and walked on a treadmill for 30 minutes at a moderate intensity before the second 24-hour measurement. Participants kept activity diaries and sleep records for each measurement. Repeated measures ANOVA was used to assess group differences across conditions.

RESULTS: The condition effect was not significant $(p=.30)$ and the group by condition interaction approached significance ( $p=.08)$. The group effect was significant $(\mathrm{p}=.009)$. This difference was characterized by greater dipping in the normotensive and dipper prehypertensive participants compared to ND prehypertensive participants. Although the interaction effect was not significant, ND prehypertensive participants were the only group to experience greater MAP dipping after acute exercise.

CONCLUSION: Our results indicate that the ND pattern for MAP can be improved with moderate intensity acute exercise in ND women at increased risk for developing hypertension.

Supported by: College of William \& Mary Summer Research Grant; Borgenicht Program for Aging Studies and Exercise Science

1962 Board \#91 June $3 \quad 9: 00$ AM - 10:30 AM

Fitness Improvements In 8 And 12 Week Exercise Programs For Obese Youth

Alex Sternberg, Sc.D MPH ${ }^{1}$, Moolamannil Abraham, MD MPH${ }^{1}$, Michael Kalinski, Ph.D, FACSM², Cheryl , Murphy, BS ${ }^{1}$, Madu Rao, MD $^{1}$, Haesoon Lee, MD'. ${ }^{1}$ SUNY Downstate Medical Center, Brooklyn, NY. ${ }^{2}$ Kent State University, Kent, OH. (Sponsor: Michael Kalinkski PhD, FACSM)

Email: Alex.Sternberg@Downstate.edu

(No disclosure reported)

PURPOSE: Weight management programs promoting changes in behavior through nutrition and exercise education are advocated to reverse the prevalence of obesity. The optimum length of such programs, however, remains unclear. Shorter programs may be economically attractive, but may not be sufficient to 